\title{
Thermal conduction in two-dimensional complex plasma layers
}

Cite as: Phys. Plasmas 28, 010704 (2021); https://doi.org/10.1063/5.0038078

Submitted: 18 November 2020 • Accepted: 23 December 2020 • Published Online: 13 January 2021

Sergey A. Khrapak
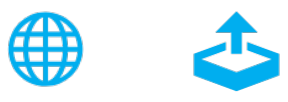

\section{ARTICLES YOU MAY BE INTERESTED IN}

Editorial: Preface to the 28th volume of Physics of Plasmas

Physics of Plasmas 28, 010401 (2021); https://doi.org/10.1063/5.0042852

ID kinetic study of pinch formation in a dense plasma focus: Transition from collisional to collisionless regimes

Physics of Plasmas 28, 010701 (2021); https://doi.org/10.1063/5.0028988

Implosion mediated gas-puff hybrid X-pinch

Physics of Plasmas 28, 010703 (2021); https://doi.org/10.1063/5.0032339 


\title{
Thermal conduction in two-dimensional complex plasma layers
}

\author{
Cite as: Phys. Plasmas 28, 010704 (2021); doi: 10.1063/5.0038078 \\ Submitted: 18 November 2020 - Accepted: 23 December 2020 . \\ Published Online: 13 January 2021

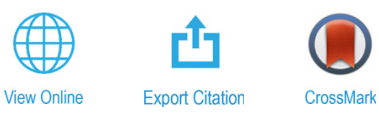

\section{Sergey A. Khrapak ${ }^{\text {a) }}$}

\section{AFFILIATIONS}

Joint Institute for High Temperatures, Russian Academy of Sciences, 125412 Moscow, Russia, Institut für Materialphysik im Weltraum, Deutsches Zentrum für Luft- und Raumfahrt (DLR), 82234 Weßling, Germany

a) Author to whom correspondence should be addressed: Sergey.Khrapak@gmx.de

\begin{abstract}
A simple vibrational model of heat transfer in two-dimensional (2D) fluids relates the heat conductivity coefficient to the longitudinal and transverse sound velocities, specific heat, and mean interatomic separation. This model is demonstrated not to contradict the available experimental and numerical data on heat transfer in $2 \mathrm{D}$ complex plasma layers. Additionally, the heat conductivity coefficient of a $2 \mathrm{D}$ onecomponent plasma with a logarithmic interaction is evaluated.
\end{abstract}

Published under license by AIP Publishing. https://doi.org/10.1063/5.0038078

Recently, a vibrational model of heat transfer in simple threedimensional (3D) liquids with soft interparticle interactions has been discussed. ${ }^{1}$ This model has been applied to quantify heat transfer in a strongly coupled one-component plasma (OCP) and Lennard-Jones fluids, and remarkable agreement with available numerical results has been reported. If there is some truth in this simple model, it can be very straightforwardly generalized to two-dimensional 2D systems. The purpose of this Letter is to present such a generalization and to compare its prediction with existing results from experiments and simulations on heat transfer in 2D complex plasma layers. Additionally, we make a prediction about the yet unknown heat conduction coefficient of a 2D OCP.

The problem of transport coefficients in $2 \mathrm{D}$ systems has been and remains a rather controversial issue. The absence of valid transport coefficients in $2 \mathrm{D}$ systems was predicted based on the analysis of the velocity autocorrelation function and of the kinetic parts of the correlation functions for the shear viscosity and the heat conductivity. ${ }^{2}$ Molecular-dynamics simulations of the $2 \mathrm{D}$ classical electron system (2D Coulomb fluid) yielded indications for the existence of a selfdiffusion coefficient. ${ }^{3}$ Strong indications of normal self-diffusion in $2 \mathrm{D}$ Yukawa fluids at sufficiently long time scales were also reported. ${ }^{4}$ The existence of finite shear viscosity coefficients of strongly coupled 2D Yukawa fluids was demonstrated in experiments with complex (dusty) plasma monolayers and molecular dynamics (MD) simulations. ${ }^{5}$ In a dedicated study of Ref. 6, it was observed that the self-diffusion coefficient exists at sufficiently high temperatures and the viscosity coefficient exists at sufficiently low temperatures, but not in the opposite limits (so that the Stokes-Einstein relation does not hold in 2D). The thermal conductivity coefficient does not appear to exist at high temperatures. For low temperatures, no definite conclusion could be drawn because of the technical challenges posed by signal-to-noise ratios and a long initial decay of the corresponding correlation function. ${ }^{6}$

An outstanding review of thermal conduction in classical lowdimensional lattices can be found in Ref. 7. The mode-coupling theory predicts the finite thermal conductivity coefficient in $3 \mathrm{D}$ and its divergence in the thermodynamic limit for lower dimensions: $\lambda \propto \ln N$ (in 2D) and $\lambda \propto N^{2 / 5}$ (in 1D), where $N$ is the number of particles in the system. Nevertheless, numerous indications in favor of the finiteness of $\lambda$ in the thermodynamic limit of low dimensional systems have also been reported over the years.

On the experimental side, finite values of the thermal conductivity coefficient were reported for single layers of crystalline, melted, and fluid complex (dusty) plasmas as measured in dedicated experiments. ${ }^{8-12}$ Numerical simulations of heat conduction in 2D Yukawa systems (which serve as a first approximation for real complex plasmas) were also performed for conditions representative to those in laboratory experiments. ${ }^{13-15}$ So, how do these results compare with theoretical expectations?

First, we generalize a simple vibrational model of heat transfer to the $2 \mathrm{D}$ situation. $2 \mathrm{D}$ fluid is approximated by a string-like structure with strings perpendicular to the temperature gradient and separated by the distance $\Delta=n^{-1 / 2}$. The average particle separation in each string is also $\Delta$. A sketch of the considered structure is shown in Fig. 1. It resembles that of a three-dimensional layered structure, but here the 


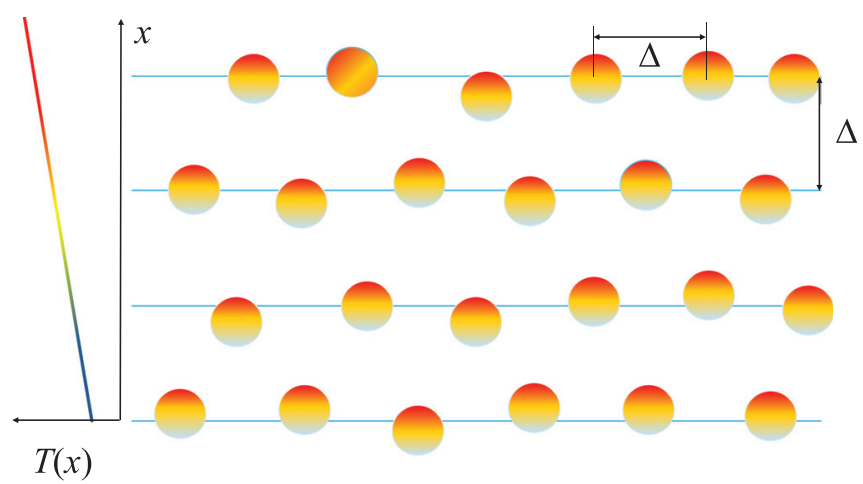

FIG. 1. Sketch of a two-dimensional fluid-like structure. The average interparticle separation is $\Delta=n^{-1 / 2}$. The temperature increases from bottom to top.

particles tend to form a disordered quasi-triangular lattice. When a temperature gradient is applied, the average difference in energy between the atoms of adjacent strings is $\Delta(d U / d x)$, where $U$ is the internal energy per particle. The energy between successive strings is transferred when two vibrating particles from adjacent strings "collide" (that is they approach by a distance that is shorter than the average interparticle separation). The characteristic energy transfer frequency is given by the average vibrational frequency of a particle, $\langle\omega\rangle / 2 \pi$. The energy flux per unit string length is $d Q / d t=-(\langle\omega\rangle / 2 \pi)(d U / d x)$, where the minus sign indicates that the heat flow is down the temperature gradient. Comparing this with Fourier's law for the heat $d Q / d t=-\lambda(d T / d x)$, where $\lambda$ is the thermal conductivity coefficient (scalar in isotropic liquids), we get

$$
\lambda=c_{V} \frac{\langle\omega\rangle}{2 \pi},
$$

where $c_{V}=d U / d T$ is the reduced heat capacity at constant volume. The temperature is measured in energy units $\left(k_{\mathrm{B}}=1\right)$, and hence, the thermal conductivity coefficient is expressed in units of frequency.

Equation (1) represents a general formula of the thermal conductivity coefficient in 2D fluids, which can be further simplified using special assumptions about the vibrational spectrum. In the simplest Einstein approximation, all particles vibrate with the same (Einstein) frequency $\Omega_{\mathrm{E}}$ (on time scales shorter than structural rearrangement timescale), and hence, $\langle\omega\rangle=\Omega_{\mathrm{E}}$. We obtain

$$
\lambda=c_{V} \frac{\Omega_{\mathrm{E}}}{2 \pi},
$$

which can be considered as a $2 \mathrm{D}$ analogue of the Horrocks and McLaughlin formula. ${ }^{16}$ Moreover, in the vicinity of the fluid-solid phase transition, we have $c_{V} \simeq 2$ to a good approximation, and hence

$$
\lambda \simeq \frac{\Omega_{\mathrm{E}}}{\pi} .
$$

This can be used as a rough estimate of the thermal conductivity coefficient of $2 \mathrm{D}$ melts.

As a more accurate approximation, we consider the conventional long-wavelength spectrum containing one longitudinal and one transverse acoustic-like modes with

$$
\omega_{l}^{2} \simeq c_{l}^{2} k^{2} ; \quad \omega_{t}^{2} \simeq c_{t}^{2} k^{2},
$$

where $c_{l}$ and $c_{t}$ are the longitudinal and transverse sound velocities. The standard averaging procedure yields

$$
\langle\omega\rangle=\frac{1}{4 \pi n} \int_{0}^{k_{\max }} k d k\left[\omega_{l}(k)+\omega_{t}(k)\right]=\frac{k_{\max }^{3}\left(c_{l}+c_{t}\right)}{12 \pi n} .
$$

The cutoff wavenumber $k_{\max }$ is chosen to provide $n$ oscillations for each collective mode, which is $\pi\left(k_{\max } / 2 \pi\right)^{2}=n$ (to ensure that we have $2 N$ normal modes for $N$ particles in $2 \mathrm{D}$ ). The average vibrational frequency becomes

$$
\langle\omega\rangle=\frac{2 \sqrt{\pi}}{3} \frac{c_{l}+c_{t}}{\Delta} \simeq 1.18 \frac{c_{l}+c_{t}}{\Delta} .
$$

This leads to the following simple approximation for the thermal conductivity coefficient of simple 2D fluids:

$$
\lambda \simeq 0.1878 c_{V} \frac{c_{l}+c_{t}}{\Delta} .
$$

If we treat the sum $c_{l}+c_{t}$ as some effective sound velocity $c_{s}$, then Eq. (7) can be considered as a $2 \mathrm{D}$ analogue of the Bridgman equation for the thermal conductivity of liquids. ${ }^{17,18}$

In arriving at (6), two additional simplifications have been employed. First, the kinetic terms were not included in acoustic dispersion relations of Eq. (4). These are numerically small at fluid densities and can be omitted. Second, the existence of a $k$-gap in the dispersion relation of the transverse collective mode is not taken into account. This " $k$-gap" implies a minimum (critical) wave number, $k_{*}$, below which transverse (shear) waves cannot propagate. It occurs in fluids both in $3 \mathrm{D}$ and $2 \mathrm{D}$. The critical wave number tends to decrease with an increase in density and a decrease in temperature (on approaching the liquid-solid phase transition). ${ }^{19-24}$ Since the contribution from the small $k$-region is not essential in the present context, the existence of the $k$-gap does not affect the obtained results explicitly (as long as $k_{*} \ll k_{\max }$, of course). At the same time, the $k$-gap itself is directly related to the heat capacity $c_{V}$, and thus, it affects the magnitude of the thermal conductivity coefficient. ${ }^{25}$

It is now possible to compare the simple expressions (2) and (7) with the available results from experiments and simulations. We start with the experiment performed by Nosenko. ${ }^{10}$ In this experiment, the heat transport in a 2D complex plasma undergoing a solid-fluid phase transition was studied. A single layer of highly charged polymer microspheres was suspended in a plasma sheath. A part of this lattice was heated by two counterpropagating focused laser beams that moved rapidly around in the lattice and provided short intense random kicks to the particles. Above a threshold, the lattice locally melted. The spatial profiles of the particle kinetic temperature were analyzed to find a thermal conductivity coefficient. For the parameters investigated, the numerical value of $\lambda \simeq 21 \mathrm{~s}^{-1}$ was obtained. ${ }^{10,26}$ All the parameters necessary for comparison with the present model were measured experimentally. The longitudinal and shear waves had the sound velocities $c_{l} \simeq 28.7 \mathrm{~mm} / \mathrm{s}$ and $c_{t} \simeq 5.4 \mathrm{~mm} / \mathrm{s}$. The interparticle separation $\Delta$, measured from the radial distribution function $g(r)$, was about $0.7 \mathrm{~mm}$ in the center of the particle layer. Substituting these numbers into Eq. (7) yields $\lambda \simeq 18.3 \mathrm{~s}^{-1}$ if $c_{V} \simeq 2$ is assumed. Actually, the specific heat $c_{V}$ is known to somewhat exceed the harmonic value 2 (in $2 \mathrm{D}$ ) near the fluid-solid phase transition due to 
anharmonic effects, both in the fluid and solid phases. ${ }^{27}$ This would shift the theoretical value even closer to the experimental result.

Next, let us consider some of the available results from numerical simulations. All simulations correspond to 2D Yukawa systems, as the first approximation of real dusty plasma layers, and some background information is necessary. 2D Yukawa systems represent point-like particles interacting via the pairwise interaction potential $\phi(r)=\left(Q^{2} / r\right) \exp \left(-r / \lambda_{\mathrm{s}}\right)$, where $Q$ is the particle charge and $\lambda_{\mathrm{s}}$ is the screening length. Equilibrium structural properties of these systems are fully characterized by the two dimensionless parameters: the coupling parameter $\Gamma=Q^{2} / a T$ and the screening parameter $\kappa=a / \lambda_{\mathrm{s}}$, where $a=(\pi n)^{-1 / 2}$ is the $2 \mathrm{D}$ Wigner-Seitz radius, $T$ is the temperature in energy units $\left(k_{\mathrm{B}}=1\right)$, and $n$ is the (areal) density. Equilibrium thermodynamic properties and the phase diagram of 2D Yukawa systems are relatively well investigated (see, e.g., Refs. 28-30). Dynamics is also affected by the damping coefficient $\gamma$, which is mainly related to the presence of neutral atoms in weakly ionized dusty plasma (and is often referred to as Epstein drag).

Hou and Piel (HP) ${ }^{13}$ conducted nonequilibrium Brownian dynamics simulations to study heat transfer in strongly coupled 2D Yukawa systems, for parameters close to those in real experiments. ${ }^{8,10}$ In HP simulation, the system was first brought to an equilibrium with a desired temperature in either a liquid or a solid state. Then, half of the system was heated to a higher temperature by applying a Gaussian white noise with desired strength. The evolution of the temperature profile and the heat flux were recorded. A steady state was approached after a substantially long period. Fitting the stationary temperature profile to a simple analytical model yielded the heat conductivity coefficient. In their simulations, the screening parameter was kept constant at $\kappa=1$, while $\Gamma$ and $\gamma$ were varied to examine different equilibrium states and different damping rates. HP reported $\lambda \simeq 0.35 \omega_{\mathrm{p}}$ for the parameters of the experiment in Ref. 10 , where $\omega_{\mathrm{p}}=\sqrt{2 \pi Q^{2} n / m a}$ is the $2 \mathrm{D}$ plasma frequency (it was observed that $\lambda$ rises slightly with the decrease in $\gamma$ and tends to $\lambda \simeq 0.4 \omega_{p}$ in the frictionless limit). From the theoretical side, we have $\Omega_{\mathrm{E}} \simeq 0.5 \omega_{\mathrm{p}}$ at $\kappa=1$ (see Fig. 4 from Ref. 31). Substituting this into Eq. (3), we get $\lambda=0.16 \omega_{\mathrm{p}}$. Alternatively, we can substitute the sound velocities $c_{l} \simeq 0.81 \omega_{\mathrm{p}} a$ and $c_{t} \simeq 0.23 \omega_{\mathrm{p}} a$ of strongly coupled 2D Yukawa fluids at $\kappa=1^{32,33}$ to obtain $\lambda \simeq 0.22 \omega_{\mathrm{p}}$. Both theoretical approximations are somewhat lower than HP numerical results.

Khrustalyov and Vaulina (KV) ${ }^{14}$ studied the thermal conductivity of equilibrium 2D Yukawa systems by means of Langevin molecular dynamics simulations. They calculated the thermal conductivity coefficient from the Green-Kubo expression. The influence of frictional dissipation on the heat transfer was investigated. They expressed the thermal conductivity coefficient (unconventionally) in units of $n \omega^{*} r_{p}^{2}$, where $\omega^{*}$ is some characteristic frequency of collisions between the charged macroparticles and $r_{p}$ is some characteristic interparticle separation. In the frictionless limit, they obtained $\lambda / n \omega^{*} r_{p}^{2} \simeq 0.86$ near the fluid-solid phase transition, independently of $\kappa$ (for $\kappa \leq 4$ ). From the information presented in Ref. 14, we can estimate $r_{p} / a$ $\simeq 1.4$ and $\omega^{*} \simeq 0.34 \omega_{\mathrm{p}}$ in the weakly screened limit $(\kappa \lesssim 1) .{ }^{34}$ This yields $\lambda \simeq 0.18 \omega_{\mathrm{p}}$, which is compatible with the theoretical approximation presented above. For example, in the weakly screening limit $\kappa \ll 1$, we have $\Omega_{\mathrm{E}} \simeq 0.6 \omega_{\mathrm{p}}$ (see Fig. 4 from Ref. 31 ). Using Eq. (3), we immediately get $\lambda=0.19 \omega_{\mathrm{p}}$.

A homogenous nonequilibrium molecular dynamics simulation was used to compute the thermal conductivity coefficient of $2 \mathrm{D}$ strongly coupled Yukawa fluids by Shahzad and He (SH). ${ }^{15}$ They used two different normalizations for $\lambda$ (by employing plasma and Einstein frequencies, respectively) and attempted to compare their results with those from earlier simulations (including 3D results) and experiments. The comparison is not conclusive: The results for $\lambda$ from HP simulation appear smaller than those from KV simulation, contrary to the actual state of affairs. Nevertheless, the results presented in Fig. 5 of Ref. 15 seem to indicate that the quantity $\lambda / n \Omega_{\mathrm{E}} a^{2}$ approaches unity as the crystallization point is approached. This finding is perfectly consistent with Eq. (3) since $\pi n a^{2}=1$.

Thus, the vibrational model of heat conductivity in $2 \mathrm{D}$ complex plasma layers is not inconsistent with the results from experiments and simulations for which comparison is possible. Except one (HP simulation) case, the model is able to reproduce closely the numerical values of the heat conductivity coefficient. In the HP case, ${ }^{13}$ the theoretical result is more than $30 \%$ lower than that from simulation, and the reason for this is unclear.

What becomes also clear after careful analysis of the available simulations is that there is an obvious deficiency of the numerical data (compared to the 3D case ${ }^{35-42}$ ). Only several state points have been investigated so far. No careful analysis of general trends and tendencies across the phase diagram has been performed. For instance, even the temperature dependence of $\lambda$ in the strongly coupled regime remains unclear (apart from the fact that it is rather weak). Conflicting predictions have been made: In Refs. 14 and 15, the data comply with a monotonous decrease in $\lambda$ on approaching the freezing point. In Ref. 13 , slight increase in $\lambda$ was suggested. The vibrational model predicts a weak increase in $\lambda$ on approaching the fluid-solid transition, mainly due to an increase in the specific heat $c_{V}$.

The dependence of $\lambda$ on the system size was not investigated systematically, probably because the interest was mainly concentrated on conditions close to those in laboratory experiments. In experiments, 5000-10 000 particles formed a 2D horizontal layer, of which positions and movements of about 900 were traced using a video camera. HP used 10000 particles in their simulation, KV varied the number of particles from 256 to 1225, and SH used $N=1024,4096,14400$, and 22500 particles but did not provide any convincing conclusions regarding the system size dependence of $\lambda$. It cannot be completely excluded that some difference between the reported results may arise from the system size effects.

Overall, relatively little is still known about thermal conductivity in 2D Yukawa systems (and about 2D systems in general). Although the vibrational model does not contradict the results reported so far, more results are needed to get more confidence regarding its applicability conditions. Experiments in large 2D complex plasma systems, ${ }^{43}$ as well as new simulations covering different regions of the phase space and addressing the effect of system size, are required to fulfill this program.

To provide one more test case for potential future comparison, we will now calculate a yet unknown coefficient of thermal conductivity of a 2D one-component plasma (OCP) with a logarithmic interaction potential. The logarithmic potential, $\phi(r)=-Q^{2} \ln (r / a)$, emerges from the solution of the $2 \mathrm{D}$ Poisson equation and represents the interaction between infinite charged filaments $\left(Q^{2}\right.$ is expressed in energy units). The point charges are immersed into a rigid neutralizing background to guarantee system stability and finite thermodynamical quantities. This system should not be confused with a system of point 


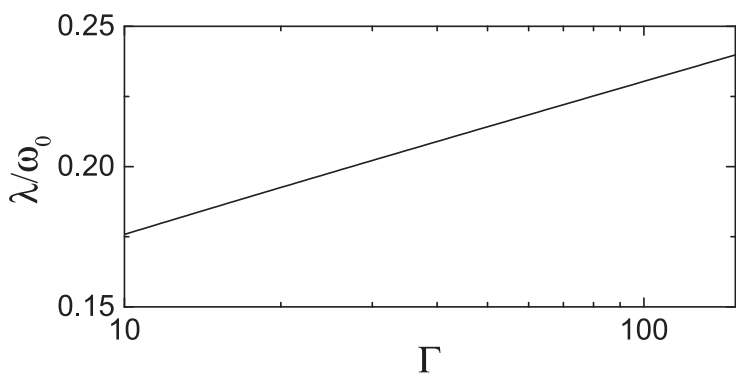

FIG. 2. Reduced thermal conductivity coefficient of a $2 \mathrm{D}$ one-component plasma with logarithmic interactions, $\lambda / \omega_{0}$, as a function of the coupling parameter $\Gamma$.

charges interacting via the conventional 3D Coulomb potential $(\propto 1 / r)$, whose motion is restricted to a $2 \mathrm{D}$ surface (which is also often referred to as OCP and represents the $\kappa=0$ limit of Yukawa systems considered above).

The thermodynamic properties of the $2 \mathrm{D}$ OCP are governed by the coupling parameter $\Gamma=Q^{2} / T$, and dynamical properties are expressed using the $2 \mathrm{D}$ frequency scale $\omega_{0}=\sqrt{2 \pi Q^{2} n / m}$. ${ }^{44-47}$ The dispersion relations of the longitudinal and transverse modes (longwavelength branch) at strong coupling can be to a good accuracy approximated by ${ }^{48}$

$$
\frac{\omega_{l, t}^{2}}{\omega_{0}^{2}}=\frac{1}{2} \pm \frac{J_{1}(q)}{q},
$$

where $q=k a$ is the normalized wavenumber, $J_{1}(x)$ is the Bessel function of the first kind, and the plus (minus) sign corresponds to the longitudinal (transverse) mode, respectively. Averaging according to Eq. (5) yields $\langle\omega\rangle \simeq 0.629 \omega_{0}$. The specific heat can be calculated from the thermal component of the internal energy, for which we use an approximation of Eq. (20) from Ref. 27. The resulting dependence of the reduced thermal conductivity coefficient on $\Gamma$ in the strongly coupled regime is shown in Fig. 2. An order of magnitude estimate can be done with the help of Eq. (3) taking into account that $\Omega_{\mathrm{E}}=\omega_{0} / \sqrt{2}$ for the $2 \mathrm{D} \mathrm{OCP}$, which results in $\lambda \simeq 0.23 \omega_{0}$ near freezing. The obtained result can be tested when numerical results on the thermal conductivity of 2D OCP become available.

To conclude, a vibrational model of heat conductivity in $2 \mathrm{D}$ fluids has been presented and discussed. It does not contradict the available results from experiments and numerical simulations of $2 \mathrm{D}$ complex plasma layers. Nevertheless, more detailed comparison is clearly warranted, and some suggestions for future research directions have been made.

I would like to thank Volodimir Nosenko for communications during the preparation of this manuscript and its careful reading.

\section{DATA AVAILABILITY}

Data sharing is not applicable to this article as no new data were created or analyzed in this study.

\section{REFERENCES}

${ }^{1}$ S. Khrapak, "Vibrational model of thermal conduction for fluids with soft interactions," Phys. Rev. E (in press).
${ }^{2}$ M. H. Ernst, E. H. Hauge, and J. M. J. van Leeuwen, "Asymptotic time behavior of correlation functions,” Phys. Rev. Lett. 25, 1254-1256 (1970).

${ }^{3}$ J. P. Hansen, D. Levesque, and J. J. Weis, "Self-diffusion in the twodimensional, classical electron gas," Phys. Rev. Lett. 43, 979-982 (1979).

${ }^{4} \mathrm{~T}$. Ott and M. Bonitz, "Is diffusion anomalous in two-dimensional Yukawa liquids?,” Phys. Rev. Lett. 103, 195001 (2009).

${ }^{5}$ Z. Donko, P. Hartmann, and J. Goree, "Shear viscosity of strongly-coupled two-dimensional Yukawa liquids: Experiment and modeling," Mod. Phys. Lett. B 21, 1357-1376 (2007).

${ }^{6} \mathrm{Z}$. Donkó, J. Goree, P. Hartmann, and B. Liu, "Time-correlation functions and transport coefficients of two-dimensional Yukawa liquids," Phys. Rev. E 79, 026401 (2009).

${ }^{7}$ S. Lepri, R. Livi, and A. Politi, "Thermal conduction in classical lowdimensional lattices," Phys. Rep. 377, 1-80 (2003).

${ }^{8}$ S. Nunomura, D. Samsonov, S. Zhdanov, and G. Morfill, "Heat transfer in a two-dimensional crystalline complex (dusty) plasma," Phys. Rev. Lett. 95, 025003 (2005).

${ }^{9}$ V. E. Fortov, O. S. Vaulina, O. F. Petrov, M. N. Vasiliev, A. V. Gavrikov, I. A. Shakova, N. A. Vorona, Y. V. Khrustalyov, A. A. Manohin, and A. V. Chernyshev, "Experimental study of the heat transport processes in dusty plasma fluid," Phys. Rev. E 75, 026403 (2007).

${ }^{10}$ V. Nosenko, S. Zhdanov, A. V. Ivlev, G. Morfill, J. Goree, and A. Piel, "Heat transport in a two-dimensional complex (dusty) plasma at melting conditions," Phys. Rev. Lett. 100, 025003 (2008).

"IJ. Goree, B. Liu, and Y. Feng, "Diagnostics for transport phenomena in strongly coupled dusty plasmas," Plasma Phys. Controlled Fusion 55, 124004 (2013).

${ }^{12}$ C.-R. Du, V. Nosenko, S. Zhdanov, H. M. Thomas, and G. E. Morfill, "Channeling of particles and associated anomalous transport in a twodimensional complex plasma crystal," Phys. Rev. E 89, 021101 (2014).

${ }^{13}$ L.-J. Hou and A. Piel, "Heat conduction in 2D strongly coupled dusty plasmas," I. Phys. A 42, 214025 (2009).

${ }^{14}$ Y. V. Khrustalyov and O. S. Vaulina, "Numerical simulations of thermal conductivity in dissipative two-dimensional Yukawa systems," Phys. Rev. E 85, 046405 (2012).

${ }^{15}$ A. Shahzad and M.-G. He, "Numerical experiment of thermal conductivity in two-dimensional Yukawa liquids," Phys. Plasmas 22, 123707 (2015).

${ }^{16} \mathrm{~J}$. K. Horrocks and E. McLaughlin, "Thermal conductivity of simple molecules in the condensed state," Trans. Faraday Soc. 56, 206 (1960).

${ }^{17} \mathrm{P}$. W. Bridgman, "The thermal conductivity of liquids under pressure," PNAAS 59, 141 (1923).

${ }^{18}$ R. B. Bird, E. N. Lightfoot, and W. E. Stewart, Transport Phenomena (Wiley, New York, 2002).

${ }^{19} \mathrm{M}$. S. Murillo, "Critical wave vectors for transverse modes in strongly coupled dusty plasmas," Phys. Rev. Lett. 85, 2514-2517 (2000).

${ }^{20}$ J. Goree, Z. Donkó, and P. Hartmann, "Cutoff wave number for shear waves and maxwell relaxation time in Yukawa liquids," Phys. Rev. E 85, 066401 (2012).

${ }^{27}$ C. Yang, M. T. Dove, V. V. Brazhkin, and K. Trachenko, "Emergence and evolution of the k-gap in spectra of liquid and supercritical states," Phys. Rev. Lett. 118, 215502 (2017).

${ }^{22}$ S. A. Khrapak, N. P. Kryuchkov, L. A. Mistryukova, A. G. Khrapak, and S. O. Yurchenko, "Collective modes of two-dimensional classical coulomb fluids," J. Chem. Phys. 149, 134114 (2018).

${ }^{23}$ S. A. Khrapak, A. G. Khrapak, N. P. Kryuchkov, and S. O. Yurchenko, "Onset of transverse (shear) waves in strongly-coupled Yukawa fluids," J. Chem. Phys. 150, 104503 (2019).

${ }^{24}$ N. P. Kryuchkov, L. A. Mistryukova, V. V. Brazhkin, and S. O. Yurchenko, "Excitation spectra in fluids: How to analyze them properly," Sci. Rep. 9, 10483 (2019).

${ }^{25}$ N. P. Kryuchkov, L. A. Mistryukova, A. V. Sapelkin, V. V. Brazhkin, and S. O. Yurchenko, "Universal effect of excitation dispersion on the heat capacity and gapped states in fluids," Phys. Rev. Lett. 125, 125501 (2020).

${ }^{26}$ V. Nosenko, private communication (2020).

${ }^{27}$ S. A. Khrapak and A. G. Khrapak, "Internal energy of the classical two- and three-dimensional one-component-plasma," Contrib. Plasma Phys. 56, 270-280 (2016). 
${ }^{28}$ P. Hartmann, G. J. Kalman, Z. Donkó, and K. Kutasi, "Equilibrium properties and phase diagram of two-dimensional Yukawa systems," Phys. Rev. E 72, 026409 (2005).

${ }^{29}$ N. P. Kryuchkov, S. A. Khrapak, and S. O. Yurchenko, "Thermodynamics of two-dimensional Yukawa systems across coupling regimes," J. Chem. Phys. 146, 134702 (2017).

${ }^{30}$ S. A. Khrapak, "Lindemann melting criterion in two dimensions," Phys. Rev. Res. 2, 012040 (2020).

${ }^{31}$ S. Khrapak and B. Klumov, "High-frequency elastic moduli of two-dimensional Yukawa fluids and solids," Phys. Plasmas 25, 033706 (2018).

${ }^{32}$ Z. Donko, G. J. Kalman, and P. Hartmann, "Dynamical correlations and collective excitations of Yukawa liquids," J. Phys.: Condens. Matter 20, 413101 (2008).

${ }^{33}$ S. A. Khrapak, "Relations between the longitudinal and transverse sound velocities in strongly coupled Yukawa fluids," Phys. Plasmas 23, 024504 (2016).

${ }^{34}$ In Ref. 14 the effective coupling parameter $\Gamma^{*}=1.5\left(Q^{2} / \operatorname{Tr}_{p}\right)(1+\kappa$ $\left.+\kappa^{2} / 2\right) e^{-\kappa}$ is used, which reaches the value $\Gamma_{c}^{*} \simeq 150$ at crystallization. This should be compared with $\Gamma=Q^{2} / T a$ and $\Gamma_{c} \simeq 140$ at crystallization in the weakly screening limit $(\kappa \ll 1) .^{27,28,30}$ Such a comparison yields $r_{p} / a \simeq 1.4$

${ }^{35}$ G. Salin and J.-M. Caillol, "Equilibrium molecular dynamics simulations of the transport coefficients of the Yukawa one component plasma," Phys. Plasmas 10, 1220-1230 (2003).

${ }^{36} \mathrm{G}$. Salin and J.-M. Caillol, "Transport coefficients of the Yukawa onecomponent plasma," Phys. Rev. Lett. 88, 065002 (2002).

${ }^{37}$ Z. Donkó and P. Hartmann, "Thermal conductivity of strongly coupled Yukawa liquids,” Phys. Rev. E 69, 016405 (2004).

${ }^{38}$ G. Faussurier and M. S. Murillo, "Gibbs-bogolyubov inequality and transport properties for strongly coupled Yukawa fluids,” Phys. Rev. E 67, 046404 (2003).
${ }^{39} \mathrm{G}$. Faussurier, "Description of strongly coupled Yukawa fluids using the variational modified hypernetted chain approach," Phys. Rev. E 69, 066402 (2004).

${ }^{40}$ A. Shahzad and M.-G. He, "Thermal conductivity calculation of complex (dusty) plasmas," Phys. Plasmas 19, 083707 (2012).

${ }^{41}$ A. Shahzad and M.-G. He, "Thermal conductivity of three-dimensional Yukawa liquids (dusty plasmas)," Contrib. Plasma Phys. 52, 667-675 (2012).

${ }^{42}$ B. Scheiner and S. D. Baalrud, "Testing thermal conductivity models with equilibrium molecular dynamics simulations of the one-component plasma," Phys. Rev. E 100, 043206 (2019).

${ }^{43}$ V. Nosenko, J. Meyer, S. K. Zhdanov, and H. M. Thomas, "New radiofrequency setup for studying large 2D complex plasma crystals," AIP Adv. 8, 125303 (2018)

${ }^{44} \mathrm{~A}$. Alastuey and B. Jancovici, "On the classical two-dimensional one-component coulomb plasma," J. Phys. 42, 1-12 (1981).

${ }^{45}$ J. M. Caillol, D. Levesque, J. J. Weis, and J. P. Hansen, "A monte carlo study of the classical two-dimensional one-component plasma," J. Stat. Phys. 28, 325-349 (1982).

${ }^{46} \mathrm{~S}$. W. de Leeuw and J. W. Perram, "Statistical mechanics of two-dimensional coulomb systems: II. The two-dimensional one-component plasma," Phys. A 113, 546-558 (1982).

${ }^{47}$ S. W. de Leeuw, J. W. Perram, and E. R. Smith, "Statistical mechanics of twodimensional coulomb systems: III. Dynamic properties of the two-dimensional one-component plasma," Phys. A 119, 441-454 (1983).

${ }^{48}$ S. A. Khrapak, B. A. Klumov, and A. G. Khrapak, "Collective modes in twodimensional one-component-plasma with logarithmic interaction," Phys. Plasmas 23, 052115 (2016). 